EPJ manuscript No.

(will be inserted by the editor)

\title{
Meson and tetra-quark mixing
}

\author{
Ping Wang ${ }^{1,2}$, Stephen R. Cotanch ${ }^{1}$ and Ignacio J. General ${ }^{3}$ \\ 1 Department of Physics, North Carolina State University, Raleigh, NC 27695-8202, USA \\ ' 2 Jefferson Laboratory, 12000 Jefferson Ave., Newport News, VA 23606, USA \\ , 3 Bayer School of Natural and Environmental Sciences, Duquesne University, Pittsburgh, PA 15282, USA
}

Received: date / Revised version: date

\begin{abstract}
The mixing between $q \bar{q}$ meson and $q \bar{q} q \bar{q}$ tetra-quark states is examined within an effective QCD Coulomb gauge Hamiltonian model. Mixing matrix elements of the Hamiltonian are computed and then diagonalized yielding an improved prediction for the low-lying $J^{P C}=0^{ \pm+}, 1^{--}$isoscalar spectra. Mixing effects were found significant for the scalar hadrons but not for the $1^{--}$states, which is consistent with the ideal mixing of vector mesons. A perturbative assessment of the exact QCD kernel is also reported.

PACS. 12.39.Mk Glueball and exotic multi-quark/gluon states - 12.39.Pn Potential models - 12.39.Ki Relativistic quark model $-12.40 . \mathrm{Yx}$ Hadron mass models and calculations
\end{abstract}

\section{Introduction}

Hadronic structure remains an interesting but challenging problem. This is because quantum chromodynamics [QCD] permits a variety of hadron formations such as $q \bar{q}$ mesons, $q \bar{q} q \bar{q}$ tetra-quarks, $q \bar{q} g$ hybrids and $g g$ glueballs. These states will, in general, also mix via quark pair annihilation/formation which further complicates this issue. Unfortunately experimental information [1] is predominantly limited to masses, widths and spectroscopic quantum numbers with little structure insight. Accordingly, theoretical input is needed and this paper reports a consistent, model study of the dynamic mixing between $q \bar{q}$ meson and $q \bar{q} q \bar{q}$ tetra-quark states.

There are abundant mixing analyses in the literature involving mesons, glueballs and hybrids utilizing several different methods including perturbation theory, relativistic Feynman-Schwinger path integrals for Green's functions [2], instantons [3], lattice QCD [4] and effective chiral approaches 5 . There have also been several mesonglueball phenomenological mixing studies [6,7, $, 8,9$ in which the meson-glueball interaction is modeled, or simply parameterized, and then diagonalized to obtain relations between primitive and physical masses.

Central to this work is the mixing between meson and tetra-quark states, which has not received much attention. The few published studies include two diquark, antidiquark cluster applications with tetra-quark mixings between either hybrid [10] or quarkonium [11] states, a mesonmeson coupled-channels scattering calculation 12 and a quark model mixing study 13 which obtained an improved scalar spectrum by adjusting phenomenological parameters.
As detailed in this paper, meson-tetra-quark mixing is fundamentally due to $q \bar{q}$ pair annihilation/formation and entails the strong confining interaction. We include this mechanism using our Coulomb gauge (CG) model, which has been successfully applied to meson, glueball, hybrid and tetra-quark states [14,15, 16, 17, 18, 19, 20,21. The model Hamiltonian is obtained from the QCD Coulomb gauge Hamiltonian using a few simplifications (see below). In this way, the original non-perturbative confining interaction can be rearranged into a calculable effective potential between color densities. We utilize powerful manybody techniques and relativistic field theory in which the non-perturbative vacuum is described as a coherent BCS ground state with quark and gluon Cooper pairs (condensates). The resulting model retains the key QCD elements and is thus capable of robust predictions as comprehensively documented in numerous publications [14, 15, 16, 17, [18, 19, 20, 21.

We only focus on the mixing between $q \bar{q}$ and $q \bar{q} q \bar{q}$ configurations and, for two reasons, omit $q \bar{q} g$ hybrid and $g g$ glueball states. First, we are interested in low mass spectra where energy level mixing arguments imply that effects from glueballs and hybrid mesons should not be large since these exotic hadrons have somewhat heavier masses. Indeed, in our previous model applications (and others including lattice results) the lightest hybrid and glueball masses are predicted to be slightly above 14,15 and below [19] $2 \mathrm{GeV}$, respectively. This is in contrast to tetra-quark masses which, due to four different color configurations, can be much lighter. In particular, we calculated [14,16] the lightest tetra-quark to be in the color singlet-singlet state with mass closer to 1 rather than $2 \mathrm{GeV}$. The other reason for omitting quark-hybrid and quark-glueball mixing matrix elements is that for our model Hamiltonian 
Ping Wang, Stephen R. Cotanch and Ignacio J. General: Meson and tetra-quark mixing

(see Section 4) the former are perturbative, and thus expected weak, while the latter entirely vanish (mixing must proceed via higher order intermediate states). This would also suggest that glueball widths might not be large, as typically expected, perhaps even narrow, consistent with a recent theoretical prediction [22. The issue of mixing involving gluonic states, however, merits a further study which we plan to address in a separate communication.

This paper is organized into seven sections. In the next section we detail the QCD Coulomb gauge Hamiltonian and then, in Section 3, present a perturbative analysis of the exact Coulomb kernel. This motivates our Coulomb gauge model Hamiltonian described in Section 4. Meson and tetra-quark mixing is treated in Section 5 with numerical results given in Section 6. Finally, key findings and conclusions are summarized in Section 7.

\section{QCD Coulomb gauge Hamiltonian}

The exact QCD Hamiltonian in the Coulomb gauge 23 is (summation over repeated indices is used throughout)

$$
\begin{aligned}
H_{\mathrm{QCD}} & =H_{q}+H_{g}+H_{q g}+H_{C} \\
H_{q} & =\int d \boldsymbol{x} \Psi^{\dagger}(\boldsymbol{x})[-i \boldsymbol{\alpha} \cdot \boldsymbol{\nabla}+\beta m] \Psi(\boldsymbol{x}) \\
H_{g} & =\frac{1}{2} \int d \boldsymbol{x}\left[\mathcal{J}^{-1} \boldsymbol{\Pi}^{a}(\boldsymbol{x}) \cdot \mathcal{J} \boldsymbol{\Pi}^{a}(\boldsymbol{x})+\mathbf{B}^{a}(\boldsymbol{x}) \cdot \mathbf{B}^{a}(\boldsymbol{x})\right](3) \\
H_{q g} & =g \int d \boldsymbol{x} \mathbf{J}^{a}(\boldsymbol{x}) \cdot \mathbf{A}^{a}(\boldsymbol{x}) \\
H_{C} & =-\frac{g^{2}}{2} \int d \boldsymbol{x} d \boldsymbol{y} \mathcal{J}^{-1} \rho^{a}(\boldsymbol{x}) K^{a b}(\boldsymbol{x}, \boldsymbol{y}) \mathcal{J} \rho^{b}(\boldsymbol{y})
\end{aligned}
$$

where $g$ is the QCD coupling constant, $\Psi$ is the quark field with current quark mass $m, A^{a}=\left(\mathbf{A}^{a}, A_{0}^{a}\right)$ are the gluon fields satisfying the transverse gauge condition, $\boldsymbol{\nabla} \cdot \mathbf{A}^{a}=0$ $(a=1,2, \ldots 8), \boldsymbol{\Pi}^{a}=-\mathbf{E}_{t r}^{a}$ are the conjugate momenta and

$$
\begin{aligned}
\mathbf{E}_{t r}^{a} & =-\dot{\mathbf{A}}^{a}+g\left(1-\nabla^{-2} \nabla \nabla \cdot\right) f^{a b c} A_{0}^{b} \mathbf{A}^{c} \\
\mathbf{E}^{a} & =-\dot{\mathbf{A}}^{a}-\nabla A_{0}^{a}+g f^{a b c} A_{0}^{b} \mathbf{A}^{c} \\
\mathbf{B}^{a} & =\nabla \times \mathbf{A}^{a}+\frac{1}{2} g f^{a b c} \mathbf{A}^{b} \times \mathbf{A}^{c}
\end{aligned}
$$

are the non-abelian chromodynamic fields. The color densities, $\rho^{a}(\boldsymbol{x})$, and quark currents, $\mathbf{J}^{a}$, are

$$
\begin{aligned}
\rho^{a}(\boldsymbol{x}) & =\Psi^{\dagger}(\boldsymbol{x}) T^{a} \Psi(\boldsymbol{x})+f^{a b c} \mathbf{A}^{b}(\boldsymbol{x}) \cdot \boldsymbol{\Pi}^{c}(\boldsymbol{x}) \\
\mathbf{J}^{a} & =\Psi^{\dagger}(\boldsymbol{x}) \boldsymbol{\alpha} T^{a} \Psi(\boldsymbol{x}),
\end{aligned}
$$

with standard $S U(3)$ color matrices, $T^{a}=\frac{\lambda^{a}}{2}$, and structure constants, $f^{a b c}$. The Faddeev-Popov determinant, $\mathcal{J}=$ $\operatorname{det}(\mathcal{M})$, of the matrix $\mathcal{M}=\boldsymbol{\nabla} \cdot \mathbf{D}$ with covariant derivative $\mathbf{D}^{a b}=\delta^{a b} \boldsymbol{\nabla}-g f^{a b c} \mathbf{A}^{c}$, is a measure of the gauge manifold curvature and the kernel in Eq. (5) is given by $K^{a b}(\boldsymbol{x}, \boldsymbol{y})=\left\langle\boldsymbol{x}, a\left|\mathcal{M}^{-1} \nabla^{2} \mathcal{M}^{-1}\right| \boldsymbol{y}, b\right\rangle$. The Coulomb gauge Hamiltonian is renormalizable, permits resolution of the Gribov problem, preserves rotational invariance, avoids spurious retardation corrections, aids identification of dominant, low energy potentials and introduces only physical degrees of freedom (no ghosts) 24.

The bare parton fields have the normal mode expansions (bare quark spinors $u, v$, helicity, $\lambda= \pm 1$, and color vectors $\left.\hat{\epsilon}_{\mathcal{C}=1,2,3}\right)$

$$
\begin{aligned}
\Psi(\boldsymbol{x}) & =\int \frac{d \boldsymbol{k}}{(2 \pi)^{3}} \Psi_{\mathcal{C}}(\boldsymbol{k}) e^{i \boldsymbol{k} \cdot \boldsymbol{x}} \hat{\epsilon}_{\mathcal{C}} \\
\Psi_{\mathcal{C}}(\boldsymbol{k}) & =u_{\lambda}(\boldsymbol{k}) b_{\lambda \mathcal{C}}(\boldsymbol{k})+v_{\lambda}(-\boldsymbol{k}) d_{\lambda \mathcal{C}}^{\dagger}(-\boldsymbol{k}) \\
\mathbf{A}^{a}(\boldsymbol{x}) & =\int \frac{d \boldsymbol{k}}{(2 \pi)^{3}} \frac{1}{\sqrt{2 k}}\left[\mathbf{a}^{a}(\boldsymbol{k})+\mathbf{a}^{a \dagger}(-\boldsymbol{k})\right] e^{i \boldsymbol{k} \cdot \boldsymbol{x}} \\
\boldsymbol{\Pi}^{a}(\boldsymbol{x}) & =-i \int \frac{d \boldsymbol{k}}{(2 \pi)^{3}} \sqrt{\frac{k}{2}}\left[\mathbf{a}^{a}(\boldsymbol{k})-\mathbf{a}^{a \dagger}(-\boldsymbol{k})\right] e^{i \boldsymbol{k} \cdot \boldsymbol{x}},
\end{aligned}
$$

with the Coulomb gauge transverse condition, $\boldsymbol{k} \cdot \mathbf{a}^{a}(\boldsymbol{k})=$ $(-1)^{\mu} k_{\mu} a_{-\mu}^{a}(\boldsymbol{k})=0$. Here $b_{\lambda \mathcal{C}}(\boldsymbol{k}), d_{\lambda \mathcal{C}}(-\boldsymbol{k})$ and $a_{\mu}^{a}(\boldsymbol{k})$ $(\mu=0, \pm 1)$ are the bare quark, anti-quark and gluon Fock operators, the latter satisfying the transverse commutation relations

$$
\left[a_{\mu}^{a}(\boldsymbol{k}), a_{\mu^{\prime}}^{b \dagger}\left(\boldsymbol{k}^{\prime}\right)\right]=(2 \pi)^{3} \delta_{a b} \delta^{3}\left(\boldsymbol{k}-\boldsymbol{k}^{\prime}\right) D_{\mu \mu^{\prime}}(\boldsymbol{k}),
$$

with

$$
D_{\mu \mu^{\prime}}(\boldsymbol{k})=\delta_{\mu \mu^{\prime}}-(-1)^{\mu} \frac{k_{\mu} k_{-\mu^{\prime}}}{k^{2}}
$$

\section{Perturbative expansion}

Before addressing meson and tetra-quark mixing, we first report a perturbative study of the kernel $K^{a b}(\boldsymbol{x}, \boldsymbol{y})$. Expanding in powers of $g$ yields

$$
\begin{aligned}
\mathcal{M}^{-1} \nabla^{2} \mathcal{M}^{-1}= & \nabla^{-2}+2 g \nabla^{-2} \mathcal{A} \nabla^{-2} \\
& +3 g^{2} \nabla^{-2} \mathcal{A} \nabla^{-2} \mathcal{A} \nabla^{-2}+\ldots,
\end{aligned}
$$

where $\mathcal{A}^{a b}=f^{a b c} \mathbf{A}^{c} \cdot \boldsymbol{\nabla}$. Note the first term represents the simple, long-ranged Coulomb interaction. We have perturbatively calculated the expectation value of the Hamiltonian component with this kernel for $0^{++} q \bar{q}$ states (the Faddeev-Popov terms are also included)

$$
E_{C} \equiv\left\langle\Psi^{J P C}\left|H_{C}\right| \Psi^{J P C}\right\rangle=g^{2} E_{2}^{C}+g^{4} E_{4}^{C}+g^{6} E_{6}^{C}+\ldots .
$$

In this paper all wavefunction kets, $|\psi\rangle$, have unit norm. The leading diagrams corresponding to $g^{2}, g^{4}$ and $g^{6}$ are shown in Fig. 1. The $g^{2}$ diagram contributes

$$
\begin{aligned}
E_{2}^{C} & =\int d \boldsymbol{q} d \boldsymbol{q}^{\prime} \frac{\mathcal{F}\left(\boldsymbol{q}, \boldsymbol{q}^{\prime}\right)}{\boldsymbol{p}^{2}} \\
\mathcal{F} & =\mathcal{U}_{\lambda_{1}}^{\dagger}(\boldsymbol{q}) \mathcal{U}_{\lambda_{1}^{\prime}}\left(\boldsymbol{q}^{\prime}\right) \mathcal{V}_{\lambda_{2}^{\prime}}^{\dagger}\left(\boldsymbol{q}^{\prime}\right) \mathcal{V}_{\lambda_{2}}(\boldsymbol{q}) \Phi_{\lambda_{1}^{\prime} \lambda_{2}^{\prime}}^{J P C}\left(\boldsymbol{q}^{\prime}\right) \Phi_{\lambda_{1} \lambda_{2}}^{J P C}(\boldsymbol{q})
\end{aligned}
$$

with $\boldsymbol{p}=\boldsymbol{q}-\boldsymbol{q}^{\prime}$ and $\boldsymbol{q}, \boldsymbol{q}^{\prime}$ the initial, final quark momenta. The dressed spinors, $\mathcal{U}_{\lambda}, \mathcal{V}_{\lambda}$, are BCS rotations of the bare spinors and wave function details are given in the following sections. The above integration is convergent and the numerical value for this Coulomb type interaction energy is $E_{2}^{C}=25.6 \mathrm{MeV}$. 

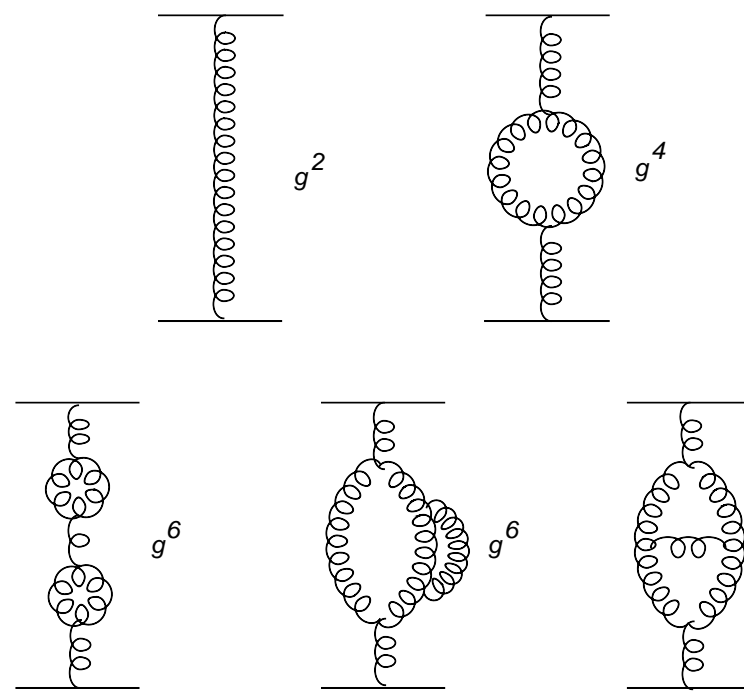

Fig. 1. Diagrams for the kernel expansion to order $g^{6}$.

The $g^{4}$ diagram reduces to

$$
E_{4}^{C}=\int d \boldsymbol{q} d \boldsymbol{q}^{\prime} d \boldsymbol{q}_{1} \frac{4\left(1-x_{1}^{2}\right) \mathcal{F}\left(\boldsymbol{q}, \boldsymbol{q}^{\prime}\right)}{\boldsymbol{p}^{2}\left(\boldsymbol{p}-\boldsymbol{q}_{1}\right)^{2} \omega\left(\boldsymbol{q}_{1}\right)},
$$

where $x_{1}=\hat{\boldsymbol{p}} \cdot \hat{\boldsymbol{q}}_{1}$. The momentum integration $\boldsymbol{q}_{1}$ over the loop diverges but replacing the gluon kinetic energy $\omega\left(\boldsymbol{q}_{1}\right)$ with $\omega\left(\boldsymbol{q}_{1}\right)^{1+\epsilon}$ yields a finite result, $A+B / \epsilon$, for positive $\epsilon$ which isolates the divergence. As shown in Fig. 2 , the integrated value scales as $1 / \epsilon$ for small $\epsilon$ which is consistent with dimensional renormalization. Extrapolating the intercept from the linear graph yields the infinite subtracted renormalized result $E_{4}^{C}=A=13.2 \mathrm{MeV}$. In this minimal subtraction scheme the coupling $g$ is renormalized to its physical value by absorbing the infinity.

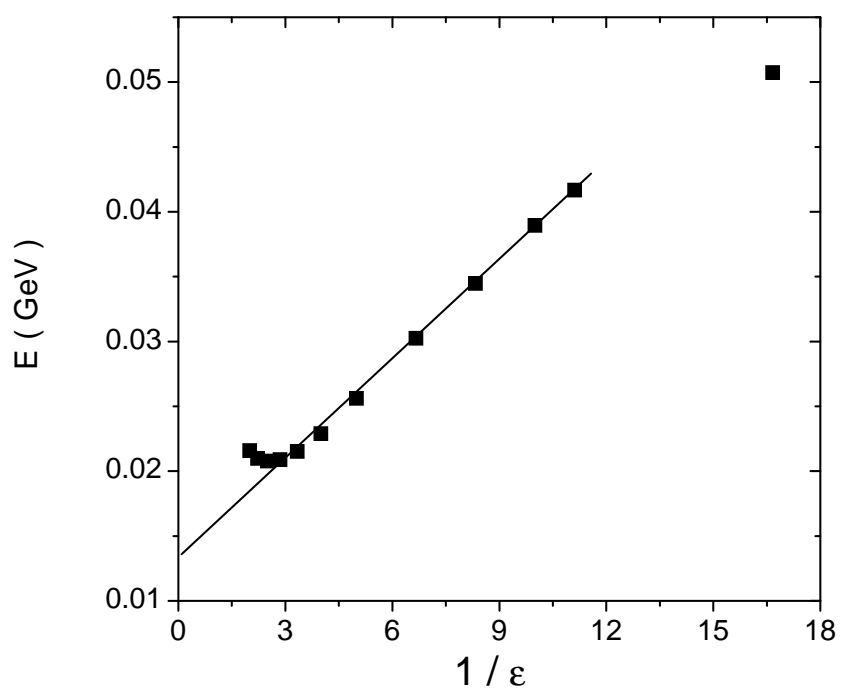

Fig. 2. The interaction energy to order $g^{4}$ versus $1 / \epsilon$.
The bottom three diagrams of Fig. 1(from left to right) are proportional to $g^{6}$, with respective expectation values

$$
\begin{gathered}
\int d \boldsymbol{q} d \boldsymbol{q}^{\prime} d \boldsymbol{q}_{1} d \boldsymbol{q}_{2} \frac{16\left(1-x_{1}^{2}\right)\left(1-x_{2}^{2}\right) \mathcal{F}\left(\boldsymbol{q}, \boldsymbol{q}^{\prime}\right)}{\boldsymbol{p}^{2}\left(\boldsymbol{p}-\boldsymbol{q}_{1}\right)^{2}\left(\boldsymbol{p}-\boldsymbol{q}_{2}\right)^{2} \omega\left(\boldsymbol{q}_{1}\right) \omega\left(\boldsymbol{q}_{2}\right)}, \\
\int d \boldsymbol{q} d \boldsymbol{q}^{\prime} d \boldsymbol{q}_{1} d \boldsymbol{q}_{2} \frac{4\left(1-x_{1}^{2}\right) \boldsymbol{k}_{1}^{2}\left(1-z^{2}\right) \mathcal{F}\left(\boldsymbol{q}, \boldsymbol{q}^{\prime}\right)}{\boldsymbol{p}^{2}\left(\boldsymbol{p}-\boldsymbol{q}_{1}\right)^{4}\left(\boldsymbol{p}-\boldsymbol{q}_{1}-\boldsymbol{q}_{2}\right)^{2} \omega\left(\boldsymbol{q}_{1}\right) \omega\left(\boldsymbol{q}_{2}\right)}, \\
\int \frac{d \boldsymbol{q} d \boldsymbol{q}^{\prime} d \boldsymbol{q}_{1} d \boldsymbol{q}_{2} \mathcal{F}\left(\boldsymbol{q}, \boldsymbol{q}^{\prime}\right)}{\boldsymbol{p}^{4}\left(\boldsymbol{p}-\boldsymbol{q}_{1}\right)^{2}\left(\boldsymbol{p}-\boldsymbol{q}_{2}\right)^{2}\left(\boldsymbol{p}-\boldsymbol{q}_{1}-\boldsymbol{q}_{2}\right)^{2} \omega\left(\boldsymbol{q}_{1}\right) \omega\left(\boldsymbol{q}_{2}\right)} \\
{\left[\boldsymbol{k}_{1} \cdot\left(\boldsymbol{k}_{1}-2 \boldsymbol{q}_{2}\right)-\boldsymbol{k}_{1} \cdot \boldsymbol{q}_{1}\left(\boldsymbol{k}_{1}-2 \boldsymbol{q}_{2}\right) \cdot \boldsymbol{q}_{1} / \boldsymbol{q}_{1}^{2}\right]} \\
{\left[\boldsymbol{k}_{2} \cdot\left(\boldsymbol{k}_{2}-2 \boldsymbol{q}_{1}\right)-\boldsymbol{k}_{2} \cdot \boldsymbol{q}_{2}\left(\boldsymbol{k}_{2}-2 \boldsymbol{q}_{1}\right) \cdot \boldsymbol{q}_{2} / \boldsymbol{q}_{2}^{2}\right]}
\end{gathered}
$$

where $z=\hat{\boldsymbol{k}}_{1} \cdot \hat{\boldsymbol{q}}_{2}, x_{j}=\hat{\boldsymbol{p}} \cdot \hat{\boldsymbol{q}}_{j}$ and $\boldsymbol{k}_{j}=2 \boldsymbol{p}-\boldsymbol{q}_{j}$ for $j=1,2$. These loop variable integrations are also divergent. Using the same renormalization procedure yields the respective values $7.15 g^{6}, 7.86 g^{6}$ and $0.48 g^{6} \mathrm{MeV}$. Therefore, the sixth order interaction energy is $E_{6}^{C}=15.5 \mathrm{MeV}$ and the series takes the form $E_{C}=\left(25.6 g^{2}+13.2 g^{4}+15.5 g^{6}\right)$ $\mathrm{MeV}$. Since the coefficients are comparable, $g^{2}$ must be less than 1, i.e. $\alpha_{s}=g^{2} / 4 \pi<0.1$, for perturbation theory to be valid but the strong interaction has $\alpha_{s}$ much larger, so the perturbative expansion fails as anticipated. We therefore seek a calculable confining kernel interaction and, guided by lattice results, adopt a linear potential specified in the next section. We note in passing that a subset or class of diagrams may still be amendable to a perturbative treatment. Specifically the chain of bubble diagrams (i.e. the $g^{4}$ and first $g^{6}$ diagrams in Fig. 1) seems to be converging. Also, ladder type diagrams (third $g^{6}$ diagram) seem much smaller, in contrast to gluon dressed or self-energy type diagrams (second $g^{6}$ diagram), and they too may be convergent. Hence further perturbative studies should be conducted to determine which parts, if any, of the exact kernel can be treated as radiative corrections and which parts are responsible for confinement and must be included non-perturbatively. This would provide further insight into the nature of confinement and also for improved QCD approximated interactions.

\section{Coulomb gauge model Hamiltonian}

Our model's starting point is the Coulomb gauge QCD Hamiltonian, Eq. (1). In this gauge, the color form of Gauss's law, which is essential for confinement, is satisfied exactly and can be used to eliminate the unphysical longitudinal gluon fields. We then make two approximations: 1) replace the exact Coulomb kernel with a calculable confining potential; 2) use the lowest order, unit value for the the Faddeev-Popov determinant. This defines the CG model Hamiltonian

$$
\begin{aligned}
& H_{\mathrm{CG}}=H_{q}+H_{g}^{\mathrm{CG}}+H_{q g}+H_{C}^{\mathrm{CG}} \\
& H_{g}^{\mathrm{CG}}=\frac{1}{2} \int d \boldsymbol{x}\left[\boldsymbol{\Pi}^{a}(\boldsymbol{x}) \cdot \boldsymbol{\Pi}^{a}(\boldsymbol{x})+\mathbf{B}^{a}(\boldsymbol{x}) \cdot \mathbf{B}^{a}(\boldsymbol{x})\right] \\
& H_{C}^{\mathrm{CG}}=-\frac{1}{2} \int d \boldsymbol{x} d \boldsymbol{y} \rho^{a}(\boldsymbol{x}) \hat{V}(|\boldsymbol{x}-\boldsymbol{y}|) \rho^{a}(\boldsymbol{y}) .
\end{aligned}
$$


Confinement is described by a kernel that is a Cornell type potential, $\hat{V}(r)=-\alpha_{s} / r+\sigma r$, where the string tension, $\sigma=0.135 \mathrm{GeV}^{2}$, and $\alpha_{s}=0.4$ have been previously determined and set the scale for the calculation.

Next, hadron states are expressed as dressed quark (anti-quark) Fock operators, $B_{\lambda_{1} \mathcal{C}_{1}}^{\dagger}\left(D_{\lambda_{2} \mathcal{C}_{2}}^{\dagger}\right)$, acting on the Bardeen-Cooper-Schrieffer (BCS) model vacuum, $|\Omega\rangle$ (see Refs. 15,17] for full details). For the tetra-quark system, the quark (anti-quark) $\mathrm{cm}$ momenta are $\mathbf{q}_{\mathbf{1}}, \mathbf{q}_{\mathbf{3}}\left(\mathbf{q}_{\mathbf{2}}, \mathbf{q}_{\mathbf{4}}\right)$ and the following wave function ansatz is adopted

$$
\begin{gathered}
\left|\Psi^{J P C}\right\rangle=\int \frac{d \boldsymbol{q}_{1}}{(2 \pi)^{3}} \frac{d \boldsymbol{q}_{2}}{(2 \pi)^{3}} \frac{d \boldsymbol{q}_{3}}{(2 \pi)^{3}} \Phi_{\lambda_{1} \lambda_{2} \lambda_{3} \lambda_{4}}^{J P C}\left(\boldsymbol{q}_{1}, \boldsymbol{q}_{2}, \boldsymbol{q}_{3}\right) \\
R_{\mathcal{C}_{3} \mathcal{C}_{4}}^{\mathcal{C}_{1} \mathcal{C}_{2}} B_{\lambda_{1} \mathcal{C}_{1}}^{\dagger}\left(\boldsymbol{q}_{1}\right) D_{\lambda_{2} \mathcal{C}_{2}}^{\dagger}\left(\boldsymbol{q}_{2}\right) B_{\lambda_{3} \mathcal{C}_{3}}^{\dagger}\left(\boldsymbol{q}_{3}\right) D_{\lambda_{4} \mathcal{C}_{4}}^{\dagger}\left(\boldsymbol{q}_{4}\right)|\Omega\rangle .
\end{gathered}
$$

The expression for the matrix $R_{\mathcal{C}_{3} \mathcal{C}_{4}}^{\mathcal{C}_{1} \mathcal{C}_{2}}$ depends on the specific color scheme selected [14,16]. Here, we focus on the color singlet-singlet scheme, $\left[(3 \otimes \overline{3})_{1} \otimes(3 \otimes \overline{3})_{1}\right]_{1}$, where the $q \bar{q}$ pairs couple to color singlets, since it gives the lowest mass among the four color representations. This yields $R_{\mathcal{C}_{3} \mathcal{C}_{4}}^{\mathcal{C}_{1} \mathcal{C}_{2}}=\delta_{\mathcal{C}_{1} \mathcal{C}_{2}} \delta_{\mathcal{C}_{3} \mathcal{C}_{4}}$. The spin part of the wave function is, $\left\langle\frac{1}{2} \frac{1}{2} \lambda_{1} \lambda_{2} \mid s_{A} \lambda_{A}\right\rangle\left\langle\frac{1}{2} \frac{1}{2} \lambda_{3} \lambda_{4} \mid s_{B} \lambda_{B}\right\rangle\left\langle s_{A} s_{B} \lambda_{A} \lambda_{B} \mid J \lambda_{A}+\lambda_{B}\right\rangle$, a product of Clebsch-Gordan coefficients. Here $J$ is the total angular momentum, $s_{A}=s_{1}+s_{2}, s_{B}=s_{3}+s_{4}$, and for scalar hadrons all orbital angular momenta, $l_{X}$, are zero, consistent with the lowest energy state (see Section 6 for p-wave pseudo-scalar and vector hadrons). A Gaussian radial wavefunction is used (see [16 for details)

$$
f\left(q_{A}, q_{B}, q_{I}\right)=e^{-\frac{q_{A}^{2}}{\alpha_{A}^{2}}-\frac{q_{B}^{2}}{\alpha_{B}^{2}}-\frac{q_{I}^{2}}{\alpha_{I}^{2}}},
$$

with variational parameters $\alpha_{A}=\alpha_{B}$ and $\alpha_{I}$ determined by minimizing the tetra-quark masses

$$
\begin{aligned}
M_{J P C} & =\left\langle\Psi^{J P C}\left|H_{\mathrm{CG}}\right| \Psi^{J P C}\right\rangle \\
& =M_{\text {self }}+M_{q q}+M_{\bar{q} \bar{q}}+M_{q \bar{q}}+M_{\text {annih }},
\end{aligned}
$$

which were previously calculated [14]16]. The subscripts indicate the source of each contribution: the $q$ and $\bar{q}$ selfenergy, the $q q, \bar{q} \bar{q}$ and $q \bar{q}$ scattering, and the $q \bar{q}$ annihilation, respectively. Finally, the $q \bar{q}$ meson state is

$$
\left|\Psi^{J P C}\right\rangle=\int \frac{d \boldsymbol{k}}{(2 \pi)^{3}} \Phi_{\lambda_{1} \lambda_{2}}^{J P C}(\boldsymbol{k}) B_{\lambda_{1}}^{\dagger}(\boldsymbol{k}) D_{\lambda_{2}}^{\dagger}(-\boldsymbol{k})|\Omega\rangle .
$$

\section{Meson and tetra-quark mixing}

In this section, we discuss the meson and tetra-quark mixing for the $J^{P C}=0^{ \pm+}$and $1^{--}$states. As discussed above, only mixing between flavored $(u, d, s) q \bar{q}$ mesons and tetra-quarks is investigated. Using the notation, $\mid q \bar{q}>$ and $\mid q \bar{q} q \bar{q}>$ for $\mid \Psi^{J P C}>$, the mixed state is given by

$$
\left|J^{P C}\right\rangle=a|n \bar{n}\rangle+b|s \bar{s}\rangle+c|n \bar{n} n \bar{n}\rangle+d|n \bar{n} s \bar{s}\rangle,
$$

where $n \bar{n}=\frac{1}{\sqrt{2}}(u \bar{u}+d \bar{d})$. The state $|s \bar{s} s \bar{s}\rangle$ is not included since its mass is much higher than the meson masses. The
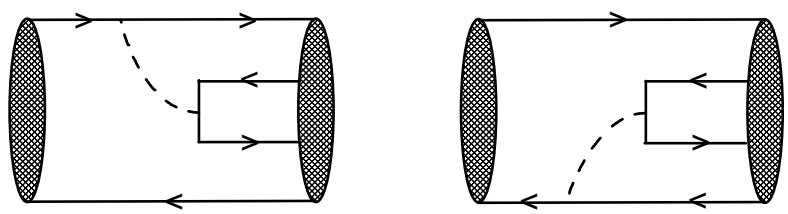

Fig. 3. Diagrams for the meson, tetra-quark mixing term.

coefficients $a, b, c$ and $d$ are determined by diagonalizing the Hamiltonian matrix, in which the meson-tetra-quark off-diagonal mixing element is (only $H_{C}^{\mathrm{CG}}$ contributes)

$$
M=\left\langle q \bar{q}\left|H_{C}^{\mathrm{CG}}\right| q \bar{q} q \bar{q}\right\rangle,
$$

where $|q \bar{q}\rangle$ is $|n \bar{n}\rangle$ or $|s \bar{s}\rangle$, and $|q \bar{q} q \bar{q}\rangle$ is $|n \bar{n} n \bar{n}\rangle$ or $|n \bar{n} s \bar{s}\rangle$. There are six off-diagonal matrix elements however two, $\left\langle s \bar{s}\left|H_{C}^{\mathrm{CG}}\right| n \bar{n}\right\rangle$ and $\left\langle s \bar{s}\left|H_{C}^{\mathrm{CG}}\right| n \bar{n} n \bar{n}\right\rangle$, vanish and one (see below), $\left\langle n \bar{n} n \bar{n}\left|H_{C}^{\mathrm{CG}}\right| n \bar{n} s \bar{s}\right\rangle$, is computed very small. The remaining mixing matrix elements are, $\left\langle n \bar{n}\left|H_{C}^{\mathrm{CG}}\right| n \bar{n} n \bar{n}\right\rangle$, $\left\langle n \bar{n}\left|H_{C}^{\mathrm{CG}}\right| n \bar{n} s \bar{s}\right\rangle$ and $\left\langle s \bar{s}\left|H_{C}^{\mathrm{CG}}\right| n \bar{n} s \bar{s}\right\rangle$. For our model Hamiltonian, there are two types of mixing diagrams illustrated in Fig. 3. Because of color factors, nonzero mixing only exists for $q \bar{q}$ annihilation between different singlet $q \bar{q}$ clusters. The expression for the first diagram in Fig. 3 is

$$
\begin{aligned}
& M_{1}=\frac{1}{2} \int d \boldsymbol{q}_{1} d \boldsymbol{q}_{2} d \boldsymbol{q}_{3} V(k) \mathcal{U}_{\lambda_{1}}^{\dagger}\left(\boldsymbol{q}_{1}\right) \mathcal{U}_{\lambda_{1}^{\prime}}\left(-\boldsymbol{q}_{4}\right) \\
& \mathcal{U}_{\lambda_{3}}^{\dagger}\left(\boldsymbol{q}_{3}\right) \mathcal{V}_{\lambda_{2}}\left(\boldsymbol{q}_{2}\right) \Phi_{\lambda_{1} \lambda_{2} \lambda_{3} \lambda_{4}}^{J P C \dagger}\left(\boldsymbol{q}_{1}, \boldsymbol{q}_{2}, \boldsymbol{q}_{3}\right) \Phi_{\lambda_{1}^{\prime} \lambda_{4}}^{J P C}\left(-2 \boldsymbol{q}_{4}\right),
\end{aligned}
$$

with $\boldsymbol{q}_{4}=-\boldsymbol{q}_{1}-\boldsymbol{k}, \boldsymbol{k}=\boldsymbol{q}_{2}+\boldsymbol{q}_{3}$ and dressed, BCS spinors

$$
\begin{gathered}
\mathcal{U}_{\lambda}=\frac{1}{\sqrt{2}}\left(\begin{array}{l}
\sqrt{1+\sin \phi(q)} \\
\sqrt{1-\sin \phi(q)}
\end{array}\right) \chi_{\lambda} \cdot \hat{\boldsymbol{q}} \\
\mathcal{V}_{\lambda}=\frac{1}{\sqrt{2}}\left(\begin{array}{ll}
-\sqrt{1-\sin \phi(q)} & \boldsymbol{\sigma} \cdot \hat{\boldsymbol{q}} \\
\sqrt{1+\sin \phi(q)}
\end{array}\right) \chi_{\lambda} .
\end{gathered}
$$

The gap angle, $\phi(q)$, is the solution to the gap equation that minimizes the energy of the BCS vacuum, i.e., the vacuum rotated by a Bogoliubov-Valatin transformation 17. The effective confining potential in momentum space is $V(k)$. The second diagram in Fig. 3 yields

$$
\begin{aligned}
& M_{2}=\frac{1}{2} \int d \boldsymbol{q}_{1} d \boldsymbol{q}_{2} d \boldsymbol{q}_{3} V(k) \mathcal{V}_{\lambda_{4}}^{\dagger}\left(\boldsymbol{q}_{4}\right) \mathcal{V}_{\lambda_{4}^{\prime}}\left(-\boldsymbol{q}_{1}\right) \\
& \mathcal{U}_{\lambda_{3}}^{\dagger}\left(\boldsymbol{q}_{3}\right) \mathcal{V}_{\lambda_{2}}\left(\boldsymbol{q}_{2}\right) \Phi_{\lambda_{1} \lambda_{2} \lambda_{3} \lambda_{4}}^{J P C \dagger}\left(\boldsymbol{q}_{1}, \boldsymbol{q}_{2}, \boldsymbol{q}_{3}\right) \Phi_{\lambda_{1} \lambda_{4}^{\prime}}^{J P C}\left(2 \boldsymbol{q}_{1}\right) .
\end{aligned}
$$

\section{Numerical results}

The two Hamiltonian parameters in our model were independently determined while the wavefunction parameters were obtained variationally. Because we seek new model masses, the unmixed variational basis states need not be ones producing a minimal, unmixed mass. Hence we can use one of the variational parameters to provide an optimal mixing prediction. We have selected $\alpha_{I}$ to exploit 


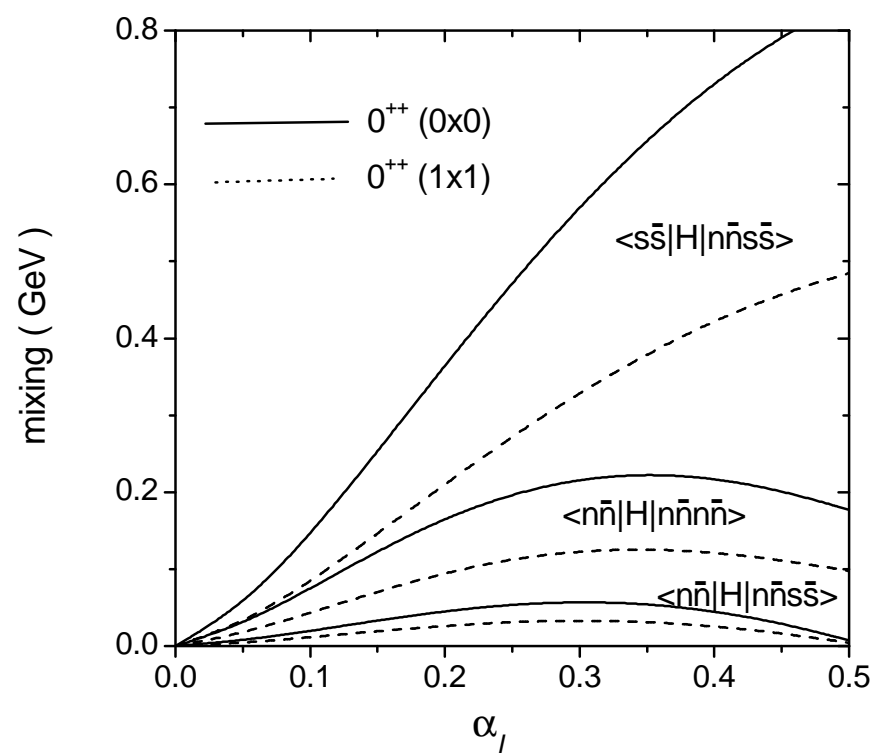

Fig. 4. The $0^{++}$mixing matrix elements versus $\alpha_{I}$. Solid and dashed lines are for $q \bar{q}$ spin 0 and 1 , respectively.

this freedom and studied the mixing sensitivity to this parameter.

The tetra-quark parity and charge parity are given by $P=(-1)^{l_{A}+l_{B}+l_{I}}$ and $C=(-1)^{l_{A}+s_{A}+l_{B}+s_{B}}$ so for the lightest, unmixed $J^{P C}=0^{ \pm+}, 1^{--}$states

$0^{++} \quad l_{A}=l_{B}=l_{I}=0, s_{A}=s_{B}=0$ or $s_{A}=s_{B}=1$,

$0^{-+} \quad l_{A}=l_{B}=0, l_{I}=1, s_{A}=s_{B}=1$,

$1^{--} \quad l_{A}=l_{B}=0, l_{I}=1, s_{A}=1$ or $s_{B}=1$.

For the $1^{--}$p-wave state we choose $l_{I}=1$ since this yields a lower mass than states with $l_{A}=1$ or $l_{B}=1$. Note for the $0^{++}$state, the spin of the two $q \bar{q}$ clusters are both either 0 or 1 and for each the three mixing matrix elements versus $\alpha_{I}$ are shown in Fig. 4. The mixing term is zero when $\alpha_{I}$ is zero and then increases with increasing $\alpha_{I}$. Also, mixing with $s \bar{s}$ states is stronger than with $n \bar{n}$ states. In particular, for $\alpha_{I}=0.2$, the $s_{A}=s_{B}=0$ matrix elements are $\left\langle s \bar{s}\left|H_{C}^{\mathrm{CG}}\right| n \bar{n} s \bar{s}\right\rangle=365 \mathrm{MeV},\left\langle n \bar{n}\left|H_{C}^{\mathrm{CG}}\right| n \bar{n} n \bar{n}\right\rangle=$ $166 \mathrm{MeV}$ and $\left\langle n \bar{n}\left|H_{C}^{\mathrm{CG}}\right| n \bar{n} s \bar{s}\right\rangle=45 \mathrm{MeV}$.

Figure 5 shows the mixing versus $\alpha_{I}$ for $0^{-+}$states. Again, all mixing terms are zero for $\alpha_{I}=0$ and then increase with increasing $\alpha_{I}$. In contrast to the $0^{++}$result, $\left\langle n \bar{n}\left|H_{\mathrm{CG}}\right| n \bar{n} n \bar{n}\right\rangle$ now has the largest value. The value $\alpha_{I}=$ 0.5 yields reasonable $\eta$ and $\eta^{\prime}$ masses with mixing elements $\left\langle n \bar{n}\left|H_{C}^{\mathrm{CG}}\right| n \bar{n} n \bar{n}\right\rangle=219 \mathrm{MeV},\left\langle n \bar{n}\left|H_{C}^{\mathrm{CG}}\right| n \bar{n} s \bar{s}\right\rangle=157 \mathrm{MeV}$ and $\left\langle s \bar{s}\left|H_{C}^{\mathrm{CG}}\right| n \bar{n} s \bar{s}\right\rangle=138 \mathrm{MeV}$.

For the $1^{--}$states a novel mixing result was obtained. The mixing matrix elements were again 0 for $\alpha_{I}=0$ but, and very interesting, also essentially 0 for all values of $\alpha_{I}$. Our model therefore predicts minimal flavor mixing for vector mesons which would explain the known $\omega / \phi$ ideal mixing. Related, weak mixing also provides a good spectrum description since the pure $n \bar{n}$ and $s \bar{s}$ states were previously in good agreement [17,18] with observation.

As mentioned above, the purely tetra-quark matrix element, $\left\langle n \bar{n} n \bar{n}\left|H_{\mathrm{CG}}\right| n \bar{n} s \bar{s}\right\rangle$, was calculated to be small since only annihilation diagrams contribute. Its value was only a few $\mathrm{MeV}$ in magnitude for any $\alpha_{I}$ and thus has no appreciable effect in this study.

With the calculated matrix elements and previously predicted unmixed meson and tetra-quark masses [14,16. 17, the complete Hamiltonian matrix was diagonalized to obtain the expansion coefficients and masses for the corresponding eigenstates. Using $\alpha_{I}=0.2$, the results for $0^{++}$ states are compared in Table 1 to the observed [1 lowest six $0^{++}$states. Noteworthy, after mixing, the $\sigma$ meson mass is shifted from $848 \mathrm{MeV}$ to $776 \mathrm{MeV}$ and the strange scalar meson mass also decreases from $1297 \mathrm{MeV}$ to $1006 \mathrm{MeV}$, now close to the experimental value of 980 $\mathrm{MeV}$. Meson and tetra-quark mixing clearly improves the model predictions as the masses of the other $f_{0}$ states are also in better agreement with data. Figure 6 illustrates the over all improved description that mixing provides for the $f_{0}$ spectrum. New structure insight has also been obtained from the coefficients, with the predictions that the $\sigma / f_{0}(600)$ is predominantly a mixture of $n \bar{n}$ and $n \bar{n} n \bar{n}$ states while the $f_{0}(980)$ consists mainly of $s \bar{s}$ and $n \bar{n} s \bar{s}$ states.

Table 2 lists the masses and coefficients for the $0^{-+}$ states for $\alpha_{I}=0.5$. Again, mixing lowers (raises) the predicted mass for states relative to unmixed $q \bar{q}(q \bar{q} q \bar{q})$ states. All mixed hadron masses are closer to measurement than the unmixed ones, except the most massive state which presumable could further mix with omitted heavier configurations. Note from the expansion coefficients that flavor mixing is again weak (i.e. $n \bar{n}$ with $s \bar{s}$ ) and less likely than meson, tetra-quark mixing having the same flavors.

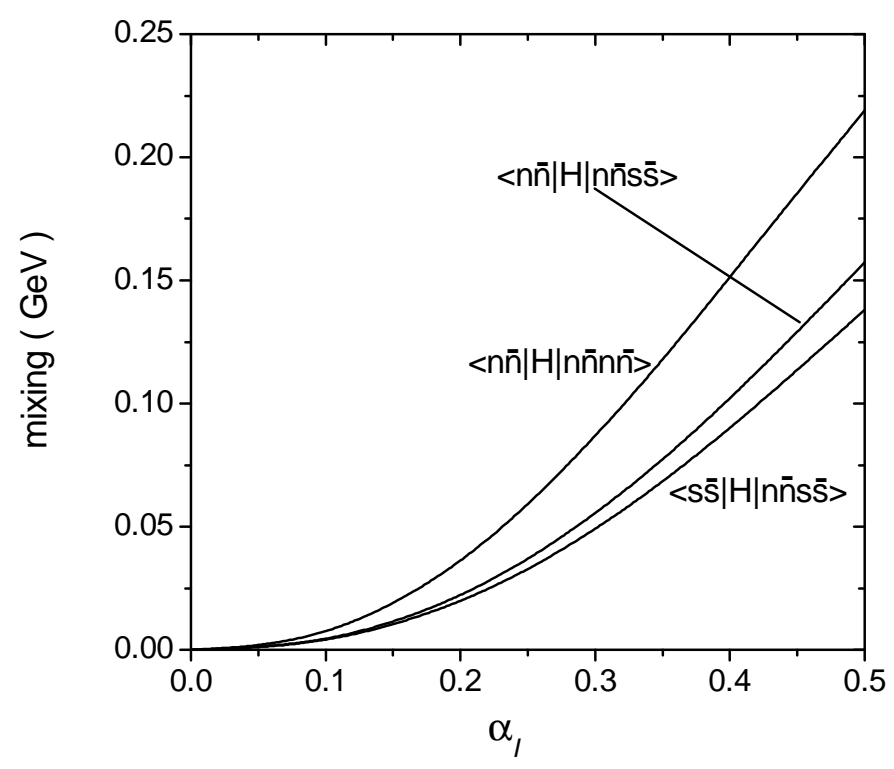

Fig. 5. The $0^{-+}$mixing matrix elements versus $\alpha_{I}$. 


\section{Summary and conclusions}

We have applied the established CG model to study $q \bar{q}$ and $q \bar{q} q \bar{q}$ mixing for the low-lying $0^{++}, 0^{-+}$and $1^{--}$spectra. In general, mixing effects are significant and provide an improved hadronic description. As important, our findings clearly document that mixing is necessary for a complete understanding of scalar and pseudo-scalar hadrons.

The mixed $0^{++}$states are a superposition of six states with coefficients obtained by diagonalizing the $H_{C G}$ Hamiltonian which decreases the mass for states dominated by $q \bar{q}$ components while increasing those predominantly having tetra-quark configurations. The resulting $f_{0}$ mass spectrum is in good agreement with observation.

Mixing is not as large for the $0^{-+}$spectrum, therefore the mass shifts are smaller. Again after mixing, predominantly $q \bar{q} q \bar{q}$ states increase in mass while the $q \bar{q}$ dominated masses decrease. All mixed states are closer to measurement except the heaviest which might be further corrected via mixing with omitted higher configurations. It is noteworthy that the CG model provides sufficient flavor mixing to produce reasonable masses for historically challenging $\eta, \eta^{\prime}$ system.

Significantly, mixing is calculated to be weak for the $1^{--}$states. Therefore, minimal flavor mixing for vector mesons follows naturally from our model, consistent with the known $\omega / \phi$ ideal mixing.

Finally, we performed a perturbative investigation of the exact QCD Coulomb gauge kernel to order $g^{6}$. As expected, a series expansion in $g$ does not converge, however a subset class of diagrams might be amendable to a perturbative treatment and further study is recommended. Future work should also address mixing applications to other

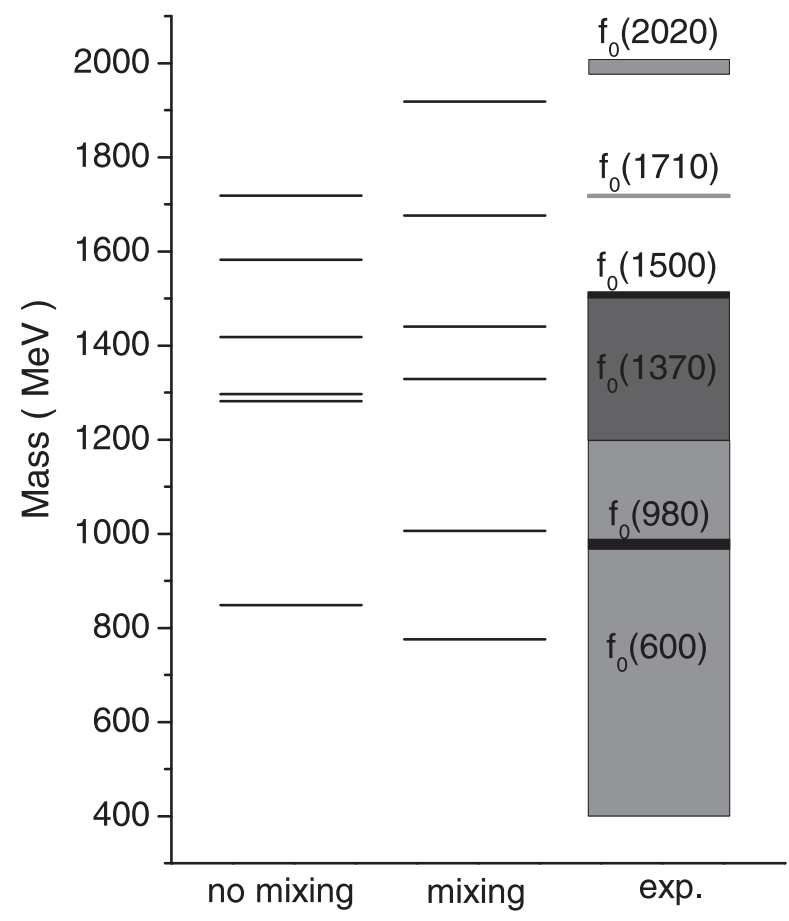

Fig. 6. Unmixed and mixed $f_{0}$ spectrum compared to data.
$I^{G}\left(J^{P C}\right)$ states, especially glueball and hybrid mesons with explicit gluonic degrees of freedom. Determining the level of mixing for these exotic systems will be important for finally establishing their existence.

Acknowledgements. The authors are very appreciative for the assistance and advice from F. J. Llanes-Estrada. Supported in part by U. S. DOE grants DE-FG02-97ER41048 and DE-FG02-03ER41260.

\section{References}

1. W.-M. Yao et al, J. Phys. G 33, 1 (2006)

2. Yu. A. Simonov, Phys. Atom. Nucl. 64, 1876 (2001)

3. N. Kochelev and D.-P. Min, Phys. Rev. D 72, 097502 (2005)

4. W.-J. Lee and D. Weingarten, Phys. Rev. D 61, 014015 (2000)

5. F. Giacosa, Th. Gutsche, V.E. Lyubovitskij and A. Faessler, Phys. Rev. D 72, 094006 (2005)

6. L. Burakovsky and P.R. Page, Eur. Phys. J. C 12, 489 (2000)

7. L.S. Celenza, B. Huang, H.S. Wang and C.M. Shakin, Brooklyn College Report No. BCCNT 99/111/283

8. F.E. Close and A. Kirk, Phys. Lett. B 483, 345 (2000)

9. D.M. Li, H. Yu and Q.-X. Shen, Commun. Theor. Phys. 34, 507 (2000)

10. H. Noya and H. Nakamura, Nucl. Phys. A 692, 348c (2001)

11. F. Giacosa, Phys. Rev. D 75, 054007 (2007)

12. E van Beveren and G. Rupp, Phys. Rev. Lett. 93, 202001 (2004)

13. B. Silvestre-Brac, J. Vijande, F. Fernandez and A. Valcarce, AIP Conf. Proc. 814, 665 (2006)

14. S.R. Cotanch, I.J. General and P. Wang, Eur. Phys. J. A 31, 656 (2007)

15. I.J. General, S.R. Cotanch and F.J. Llanes-Estrada, Eur. Phys. J. C 51, 347 (2007) arXiv:hep-ph/0609115

16. I.J. General, P. Wang, S.R. Cotanch and F.J. LlanesEstrada, Phys. Lett. B 653, 216 (2007) arXiv:0707.1286

17. F.J. Llanes-Estrada and S.R. Cotanch, Nucl. Phys. A 697, $303(2002)$

18. F.J. Llanes-Estrada, S.R. Cotanch, A.P. Szczepaniak and E.S. Swanson, Phys. Rev. C 70, 035202 (2004)

19. F.J. Llanes-Estrada, P. Bicudo and S.R. Cotanch, Phys. Rev. Lett. 96, 081601 (2006)

20. F.J. Llanes-Estrada and S.R. Cotanch, Phys. Rev. Lett. 84, $1102(2000)$

21. F.J. Llanes-Estrada and S.R. Cotanch, Phys. Lett. B 504, 15 (2001)

22. P. Bicudo, S.R. Cotanch, F.J. Llanes-Estrada and D.G. Robertson, Eur. Phys. J. C 52, 363 (2007)

23. T.D. Lee, Particle Physics and Introduction to Field Theory (Harwood Academic Publishers, New York, 1990)

24. D. Zwanziger, Nucl. Phys. B 485, 185 (1997) 
Ping Wang, Stephen R. Cotanch and Ignacio J. General: Meson and tetra-quark mixing

Table 1. Mixing coefficients and masses in $\mathrm{MeV}$ for $0^{++}$states.

\begin{tabular}{ccccccc}
\hline \hline & $\mid n \bar{n}>$ & $\mid s \bar{s}>$ & $\mid n \bar{n} n \bar{n}>_{1}$ & $\mid n \bar{n} n \bar{n}>_{2}$ & $\mid n \bar{n} s \bar{s}>_{1}$ & $\mid n \bar{n} s \bar{s}>_{2}$ \\
\hline no mixing & 848 & 1297 & 1282 & 1418 & 1582 & 1718 \\
mixing & 776 & 1006 & 1329 & 1440 & 1676 & 1918 \\
exp. & $f_{0}(600)$ & $f_{0}(980)$ & $f_{0}(1370)$ & $f_{0}(1500)$ & $f_{0}(1710)$ & $f_{0}(2020)$ \\
& $400-1200$ & $980 \pm 10$ & $1200-1500$ & $1507 \pm 5$ & $1718 \pm 2$ & $1992 \pm 16$ \\
\hline coeff. & $\mathrm{a}$ & $\mathrm{b}$ & $c_{1}$ & $c_{2}$ & $d_{1}$ & $d_{2}$ \\
$f_{0}(600)$ & 0.936 & -0.075 & 0.263 & 0.216 & 0.030 & -0.007 \\
$f_{0}(980)$ & 0.057 & 0.818 & -0.022 & -0.017 & 0.549 & -0.156 \\
$f_{0}(1370)$ & -0.308 & 0.045 & 0.922 & 0.228 & 0.008 & -0.003 \\
$f_{0}(1500)$ & -0.139 & 0.017 & -0.282 & 0.949 & 0.006 & -0.003 \\
$f_{0}(1710)$ & -0.031 & -0.240 & -0.001 & -0.002 & 0.582 & 0.776 \\
$f_{0}(2020)$ & -0.063 & -0.514 & -0.002 & -0.006 & 0.599 & -0.610 \\
\hline \hline
\end{tabular}

Table 2. Mixing coefficients and masses in $\mathrm{MeV}$ for $0^{-+}$states.

\begin{tabular}{ccccc}
\hline \hline & $n \bar{n}$ & $s \bar{s}$ & $|n \bar{n} n \bar{n}\rangle$ & $|n \bar{n} s \bar{s}\rangle$ \\
\hline no mixing & 610 & 1002 & 1252 & 1552 \\
mixing & 531 & 970 & 1316 & 1598 \\
exp. & $\eta$ & $\eta^{\prime}$ & $\eta(1295)$ & $\eta(1405)$ \\
& $547.51 \pm 0.18$ & $957.78 \pm 0.14$ & $1294 \pm 4$ & $1409.8 \pm 2.5$ \\
\hline coeff. & $\mathrm{a}$ & $\mathrm{b}$ & $\mathrm{c}$ & $\mathrm{d}$ \\
$\eta$ & 0.951 & -0.046 & 0.279 & 0.126 \\
$\eta^{\prime}$ & 0.032 & 0.973 & -0.046 & 0.223 \\
$\eta(1295)$ & -0.289 & 0.036 & 0.953 & 0.080 \\
$\eta(1405)$ & -0.108 & -0.222 & -0.105 & 0.963 \\
\hline \hline
\end{tabular}

